\title{
AARC Clinical Practice Guideline: Management of Adult Patients with Tracheostomy in the Acute Care Setting
}

\author{
Constance C Mussa, Dina Gomaa, Daniel D Rowley, Ulrich Schmidt, Emily Ginier, and \\ Shawna L Strickland
}

\author{
Introduction \\ Committee Composition \\ Search Strategy \\ Study Selection \\ Assessment and Recommendations \\ Development of Recommendations \\ Tracheostomy Bundle \\ Tracheostomy Teams \\ Protocol-Directed Care \\ Summary
}

Management of patients with a tracheostomy tube includes many components of care provided by clinicians from various health care disciplines. In recent years, clinicians worldwide have demonstrated a renewed interest in the management of patients with tracheostomy due to the recognition that more effective and efficient management of this patient population is necessary to decrease morbidity and mortality and to optimize the value of the procedure. Commensurate with the goal of enhancing the care of patients with tracheostomy, we conducted a systematic review to facilitate the development of recommendations relevant to the care of adult patients with tracheostomy in the acute care setting. From our systematic review, clinical practice guidelines were developed to address questions regarding the impact of tracheostomy bundles, tracheostomy teams, and protocol-directed care on time to decannulation, length of stay, tracheostomy-related cost, tracheostomy-related adverse events, and other tracheostomy-related outcomes in tracheostomized adult patients in the acute care setting. Using a modification of the RAND/UCLA Appropriateness Method, 3 recommendations were developed to assist clinicians with tracheostomy management of adult patients in the acute care setting: (1) evidence supports the use of tracheostomy bundles that have been evaluated and approved by a team of individuals experienced in tracheostomy management to decrease time to decannulation, tracheostomyrelated adverse events, and other tracheostomy-related outcomes, namely, improved tolerance of oral diet; (2) evidence supports the addition of a multidisciplinary tracheostomy team to improve time to decannulation, length of stay, tracheostomy-related adverse events, and other tracheostomy-related outcomes, namely, increased speaking valve use; (3) evidence supports the use of a weaning/decannulation protocol to guide weaning and removal of the tracheostomy tube to improve time to decannulation. Key words: tracheostomy; tracheostomy care; tracheostomy team; tracheostomy bundle; tracheostomy protocol. [Respir Care 2021;66(1):156-169. (C) 2021 Daedalus Enterprises] 


\section{Introduction}

A tracheostomy is commonly performed in critically ill patients because it potentially confers several benefits over prolonged endotracheal intubation, including airway stability, facilitation of oral intake, increased comfort, and, possibly, facilitation of weaning. ${ }^{1-8}$ Tracheostomy is one of the most common surgical procedures performed in mechanically ventilated, critically ill, adult patients. Between 1993 and $2012,9.1 \%$ of adult patients $(n=1,352,432)$ requiring mechanical ventilation received a tracheostomy. ${ }^{9}$ A 2013 review of the University Health System Consortium database of patients cared for between 2002 and 2008 revealed that, of 44,124 acute respiratory failure subjects, 4,776 $(10.8 \%)$ underwent tracheostomy. ${ }^{10}$ The analysis also revealed that patients with tracheostomy, compared to patients without tracheostomy, had higher morbidity. The care of patients with tracheostomy is costlier because of their longer hospital stay and because they are commonly discharged to a long-term facility.

Halum and colleagues ${ }^{11}$ investigated the long-term outcomes of critically ill patients with ages ranging from 15 to $93 \mathrm{y}$ and reported that subjects with tracheostomy had higher mortality $(32.1 \%)$ compared with subjects who did not receive a tracheostomy $(15.4 \%)$. It has been reported that $7 \%$ of patients with tracheostomy will suffer an adverse event, and that $19.7 \%$ will not survive to discharge. ${ }^{12}$ Mehta et $\mathrm{al}^{9}$ reported that "even patients who survive the first year after tracheostomy have remarkably poor outcomes, with multiple readmissions to acute care hospitals."

Adult patients with tracheostomy consume a disproportionate amount of health care resources. ${ }^{13}$ Providing costeffective, high-quality care for patients with tracheostomies is a complex undertaking that can be challenging due to

Dr Mussa is affiliated with Point of Light Consulting, Brooklyn, New York. Ms Gomaa is affiliated with the Department of Surgery, Division of Trauma/Critical Care, University of Cincinnati, Cincinnati, Ohio. Mr Rowley is affiliated with Pulmonary Diagnostics \& Respiratory Therapy Services, University of Virginia Medical Center, Charlottesville, Virginia. Dr Schmidt is affiliated with the Department of Anesthesiology, University of California San Diego, San Diego, California. Ms Ginier is affiliated with the Taubman Health Sciences Library, University of Michigan, Ann Arbor, Michigan. Dr Strickland is affiliated with the American Association for Respiratory Care, Irving, Texas.

Supplementary material related to this paper is available at http://www. rcjournal.com.

Mr Rowley has disclosed relationships with Draeger, Philips, and Mallinckrodt. Dr Strickland is an executive staff member of the American Association for Respiratory Care. The remaining authors have disclosed no conflicts of interest.

Correspondence: Constance C Mussa PhD RRT. Email:

ccmussa@pointoflightconsulting.org.

DOI: $10.4187 /$ respcare. 08206 multiple factors, including the nature and severity of disease, patient age, the use of an extensive assortment of tracheostomy tubes, and variations in the skill level of care providers. Effective and efficient management of patients with tracheostomy is necessary to prevent morbidity and mortality and to reduce the cost of care in this patient population. We conducted a systematic review of peer-reviewed literature to develop recommendations that could enhance the care of adult patients in the acute care setting with tracheostomy tubes in situ. The clinical practice guidelines that were developed from this systematic review are centered around the following questions relevant to the management of adult patients in the acute care setting:

1. Does the use of a tracheostomy bundle impact time to decannulation, length of stay (LOS), tracheostomyrelated cost, and tracheostomy-related adverse events in tracheostomized adult patients in the acute care setting?

2. Does the use of tracheostomy teams impact time to decannulation, LOS, tracheostomy-related cost, and tracheostomy-related adverse events in tracheostomized adult patients in the acute care setting?

3. Does protocol-directed care impact time to decannulation, LOS, tracheostomy-related cost, and tracheostomy-related adverse events in tracheostomized adult patients in the acute care setting?

\section{Committee Composition}

A committee was selected by American Association for Respiratory Care (AARC) leadership based on their known experience related to the topic, interest in participating in the project, and commitment to the process details. The committee first met face-to-face, where they were introduced to the process of developing clinical practice guidelines. At that time, the committee selected a chair and wrote a first draft of questions in a format that directly related to the patient, intervention, comparison, and outcome (PICO). Subsequent meetings occurred as needed by conference call and included AARC staff as needed. Frequent email communications occurred among committee members and AARC staff. The committee members received no remuneration for their participation in the process, though their expenses for the faceto-face meeting were covered by the AARC.

\section{Search Strategy}

A literature search was conducted using the PubMed, CINAHL via EBSCOhost, and the Scopus.com databases for studies on tracheostomy care in hospitalized adult patients. The search strategies used a combination of relevant controlled vocabulary (ie, Medical Subject Headings and CINAHL Headings) and keyword variations that 
related to tracheostomy care and techniques, hospitalization, and outcomes. The searches were limited to Englishlanguage studies about human populations. The searches were also designed to filter out citations indexed as commentaries, editorials, interviews, news, or reviews. No date restrictions were applied to the searches. Refer to the online supplement for the complete search strategy executed in each database (see the supplementary materials at http:// www.rcjournal.com). Duplicate citations were identified and removed using EndNote X7 citation management software (Clarivate Analytics, Philadelphia, Pennsylvania).

\section{Study Selection}

Two reviewers independently assessed study eligibility in the Covidence systematic review software (Melbourne, Australia). Inclusion criteria used to assess eligibility were: (1) tracheostomy and (2) adult population. The exclusion criteria used were: (1) not tracheostomy care, (2) non-clinical topic, (3) pediatric population, (4) endotracheal tube, (5) intubated patients, (6) laryngectomy, (7) case study, and (8) not empirical research (eg, theory or opinion articles).

\section{Assessment and Recommendations}

The search strategies retrieved 1,457 articles. After the removal of duplicates, 1,117 articles remained for screening, of which 1,000 were excluded at the title and abstract level. Of the remaining 117 articles, 96 were excluded following full text review against the inclusion and exclusion criteria. During the extraction phase, 4 additional articles were excluded. A total of 17 articles were included in this systematic review (Fig. 1). Of these studies, 10 focused on the use of tracheostomy teams, ${ }^{14-23} 5$ focused on the use of a tracheostomy weaning/decannulation protocol, ${ }^{24-28} 1$ study focused on the use of a tracheostomy-related acquired pressure ulcer (TRAPU) bundle, ${ }^{29}$ and 1 study focused on a tracheostomy management bundle. ${ }^{30}$ Tables $1-3$ present the key details from the 17 included studies, and Table 4 provides a summary of the key findings for each PICO question addressed in this review.

Risk of bias for most of the studies (no. $=16$ ) was ranked as low or moderate at the quality assessment; only 1 study was ranked as high. The most common limitations to the quality of the studies were small sample size, retrospective study design, inadequate description of study subjects and procedures, and weakness in statistical methodology.

\section{Development of Recommendations}

It is recognized that a process is necessary to combine the best available evidence with the collective experience of committee members. To achieve this, a modification of

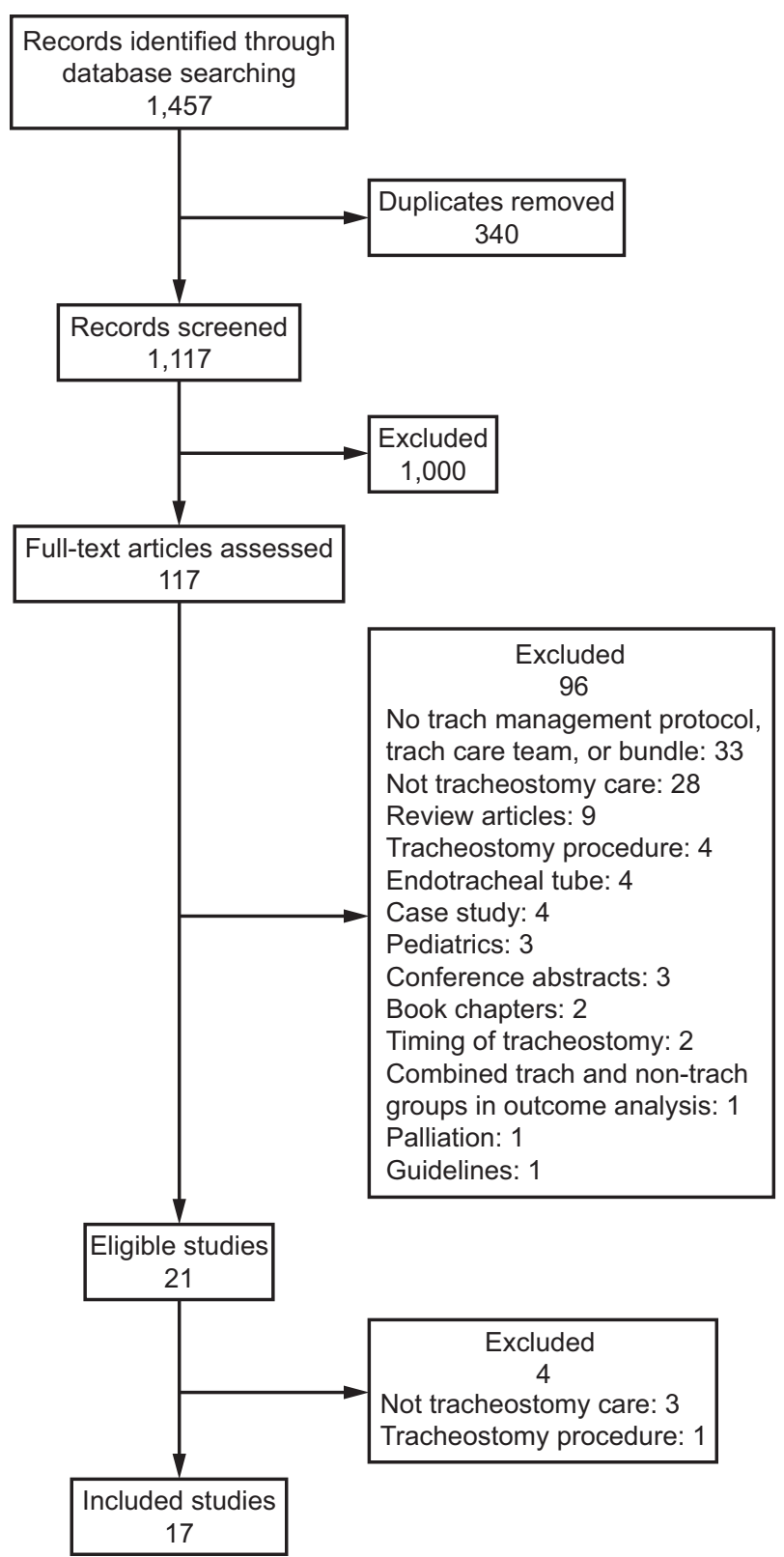

Fig. 1. Flow chart.

the RAND/UCLA Appropriateness Method $^{31}$ was used. The literature was collapsed into evidence tables according to PICO question. Individual panel members were assigned the task of writing a systematic review of the topic, drafting 1 or more recommendations, and suggesting the level of evidence supporting the recommendation: (A) convincing scientific evidence based on randomized controlled trials of sufficient rigor; (B) weaker scientific evidence based on lower levels of evidence such as cohort studies, retrospective studies, case-control studies, and cross-sectional studies; (C) based on the collective experience of the committee. 


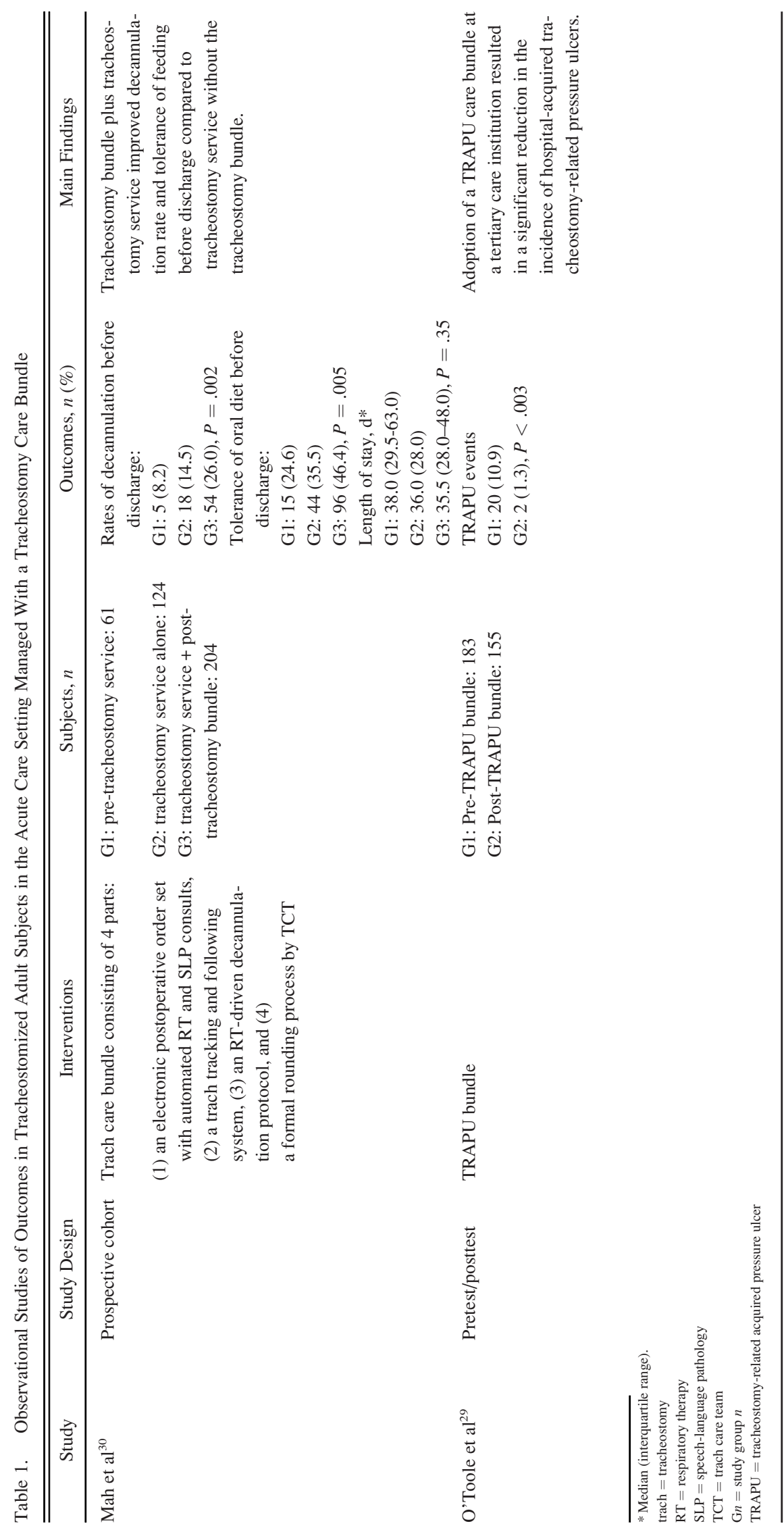




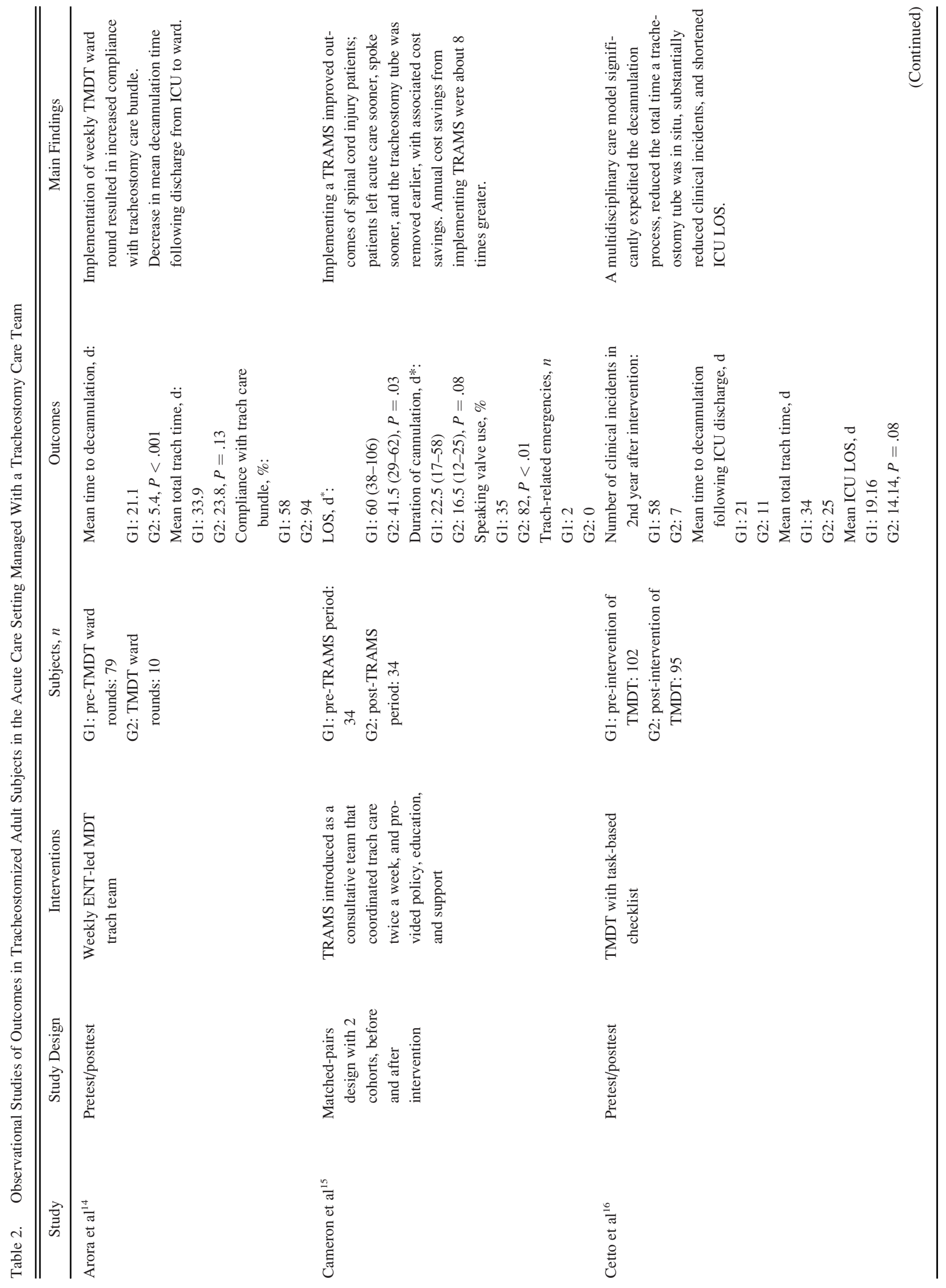




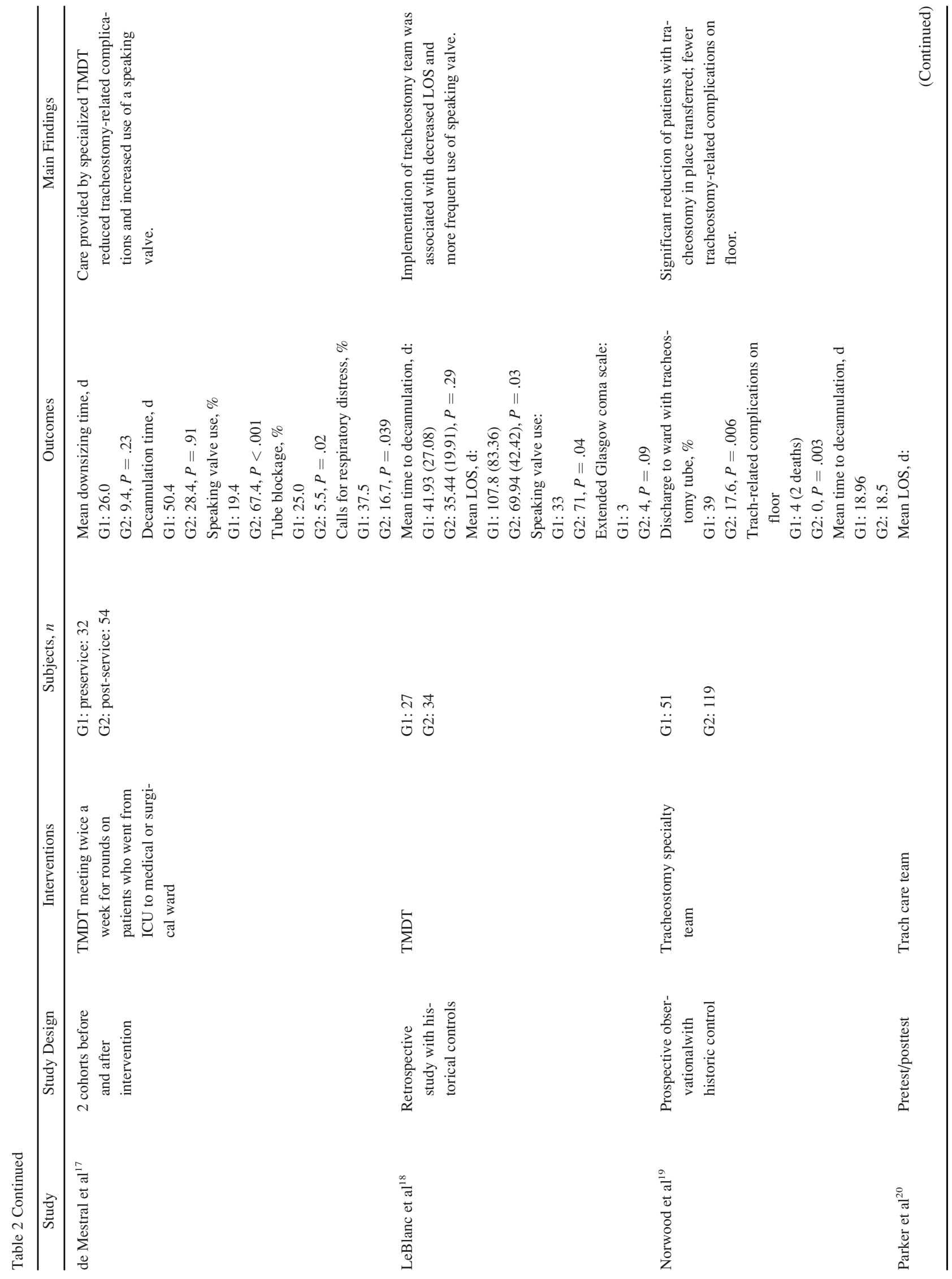



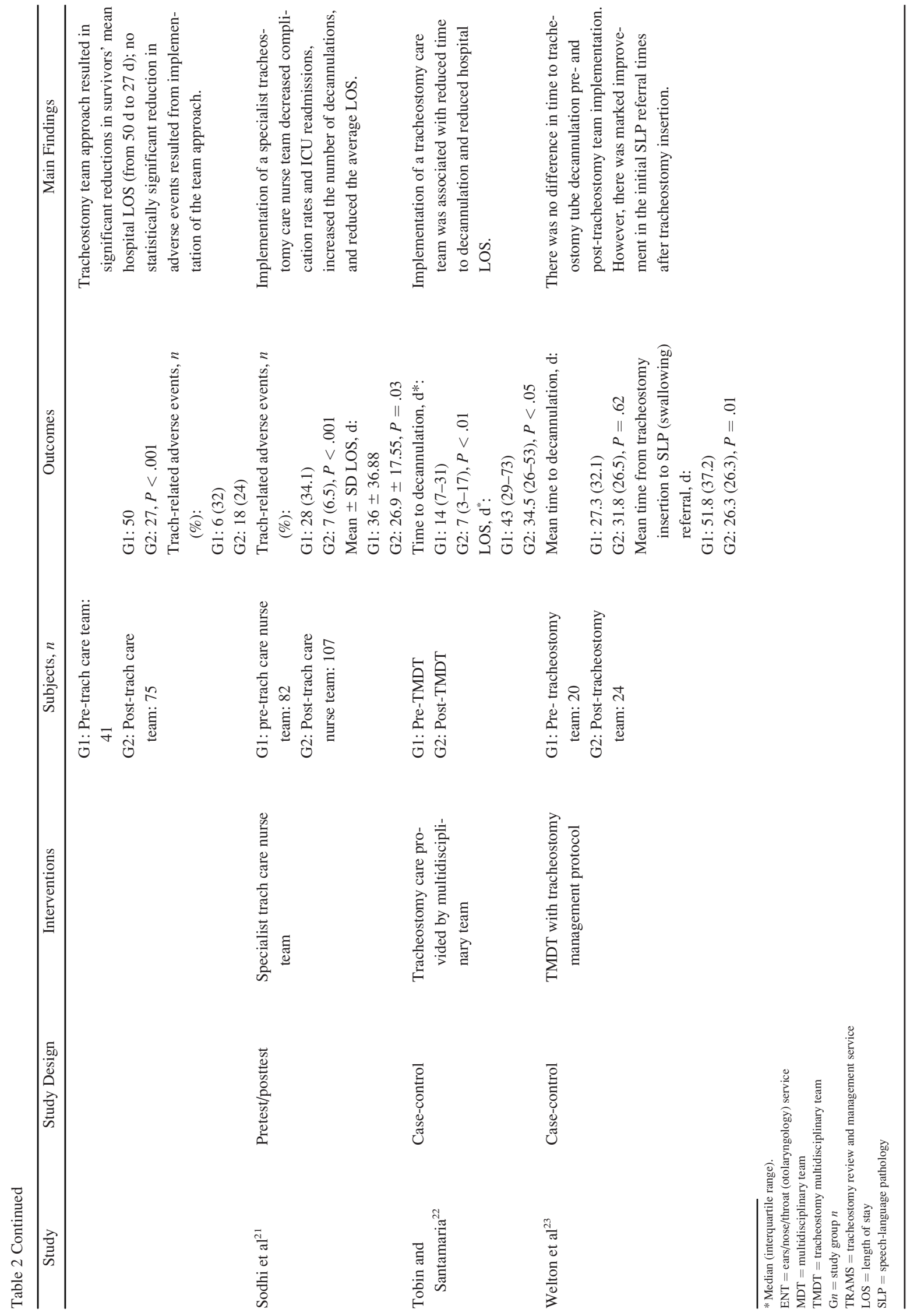


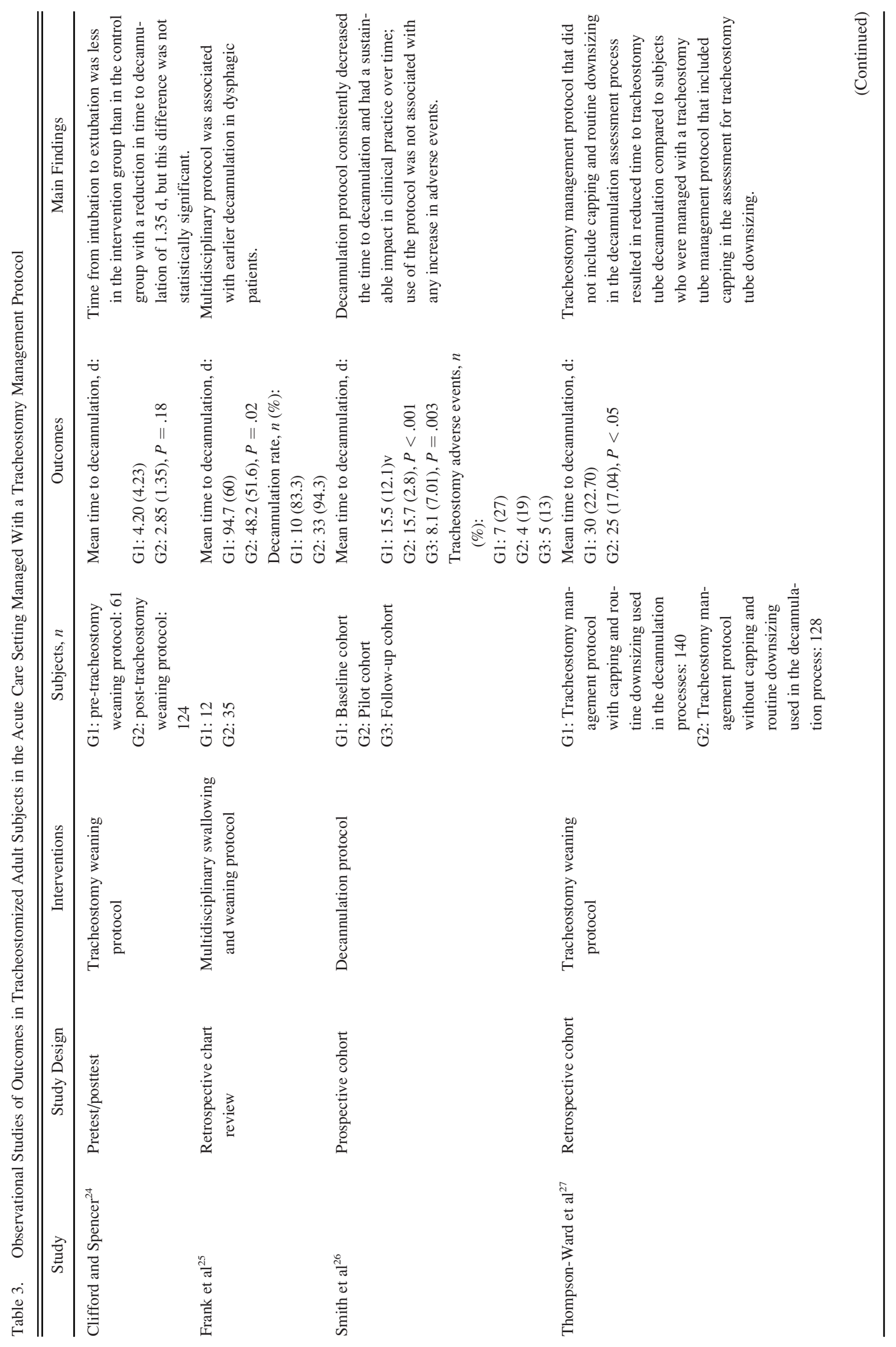




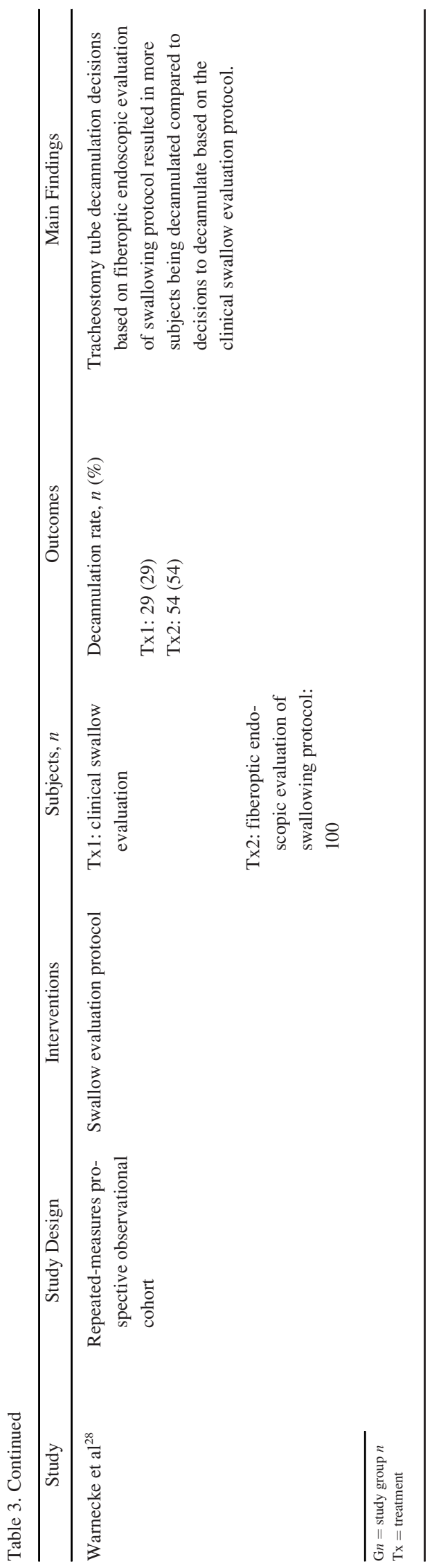

Committee members reviewed the first draft of evidence tables, systematic reviews, recommendations, and evidence levels. Each committee member rated each recommendation using a Likert scale of 1-9, with 1 meaning expected harms greatly outweigh the expected benefits and 9 meaning expected benefits greatly outweigh the expected harms. The ratings were returned to the committee chair. The first ratings were done with no interaction among committee members. A conference call was convened, during which the individual committee ratings were discussed. Particular attention was given to any outlier scores and the justification. Recommendations and evidence levels were revised with input from the committee members. After discussing each PICO question, committee members re-rated each recommendation. The final median and range of committee members' scores are reported. Strong agreement required that all committee members rank the recommendation 7 or higher, whereas weak agreement meant that one or more committee members ranked the recommendation below 7 , but the median vote was at least 7 . For recommendations with weak agreement, the percentage of committee members who rated 7 or above was calculated and reported after each weak recommendation. Figure 2 illustrates the process flow the panel used to rate the appropriateness and quality of the literature selected through the search process.

Drafts were distributed among committee members in several iterations. When all committee members were satisfied, the document was submitted for publication. The clinical practice guidelines were subjected to peer review before final publication.

\section{Tracheostomy Bundle}

Patients with tracheostomies are medically complex and require integrated care from several different health care professionals. To streamline the care of multiple providers, care bundles have been introduced. A care bundle consists of 3-5 components that describe structured approaches to providing care to a specific patient population (http://www. ihi.org/topics/bundles/pages/default.aspx, accessed June 2, 2020). Care bundles have become commonplace because their use has demonstrated improvement in both care processes and outcomes. For example, discharge care bundles for patients with COPD result in fewer hospital readmissions, ${ }^{32}$ and specific ventilator bundle components are associated with improved outcomes. $^{33}$

Despite this evidence, a review of the relevant literature yielded only 1 observational study of a tracheostomy care bundle, which consisted of 4 structured approaches to providing care for patients with tracheostomies. ${ }^{30}$ This study focused on 3 tracheostomy-related outcomes: rate of decannulation, hospital LOS, and tolerance of oral diet before discharge. 
Table 4. Summary of Recommendations for Each PICO Question

\begin{tabular}{lc}
\hline \hline \multicolumn{1}{c}{ PICO Question } & Summary of Recommendations \\
\hline $\begin{array}{l}\text { 1. Does the use of a tracheostomy bundle impact time to decannula- } \\
\text { tion, length of stay, tracheostomy-related cost, and tracheostomy- } \\
\text { related critical incidents in tracheostomized adult patients in the } \\
\text { acute care setting? }\end{array}$ & $\begin{array}{c}\text { Evidence supports the use of tracheostomy bundles that have been eval- } \\
\text { uated and approved by a team of subject-matter experts for tracheostom- } \\
\text { ized adult patients in the acute care setting (Evidence level B; median } \\
\text { appropriateness score 7, range 6-8). } \\
\text { Evidence supports the addition of a multidisciplinary tracheostomy team to } \\
\text { 2. Does the use of tracheostomy teams impact time to decannula- } \\
\text { tion, length of stay, tracheostomy-related cost, and tracheostomy- } \\
\text { related critical incidents in tracheostomized adult patients in the } \\
\text { acute care setting? }\end{array}$ \\
$\begin{array}{l}\text { 3. Does protocol-directed care impact time to decannulation, length } \\
\text { of stay, tracheostomy-related cost, and tracheostomy-related }\end{array}$ & $\begin{array}{c}\text { Evidence supports the use of a protocol to guide weaning and removal of } \\
\text { the tracheostomy tube in tracheostomized adult patients in the acute care }\end{array}$ \\
adverse events in tracheostomized adult patients in the acute care & setting (Evidence level B; median appropriateness score 8, range 8). \\
setting? & \\
\hline PICO = patient, intervention, comparison, outcome & \\
\hline
\end{tabular}

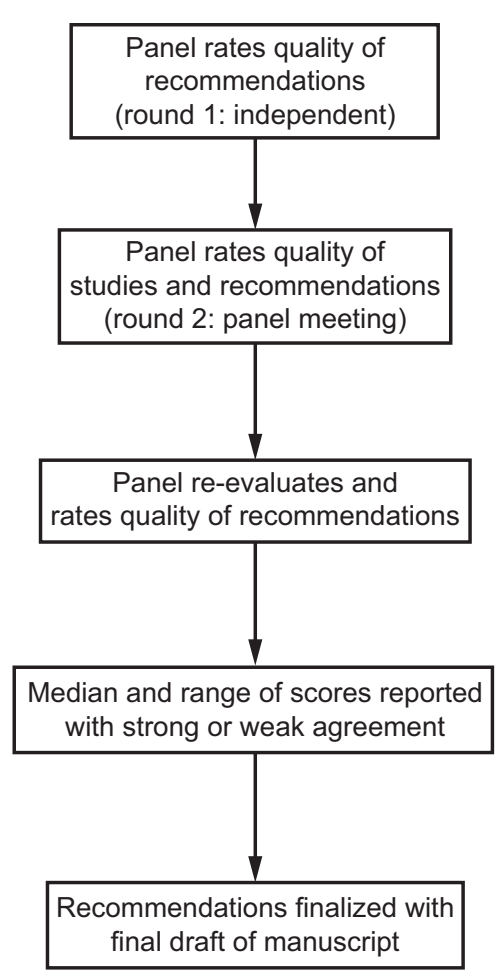

Fig. 2. Literature appraisal process.

Tracheostomy-related outcomes are important consequences of having a tracheostomy tube in situ and include time to decannulation, tracheostomy-related adverse events, tracheostomy-related health care cost, hospital LOS, tracheostomy-related pressure injury, time to oral intake, and time to communication. Only 2 studies, which were observational in nature, have addressed this question (Table 1).

Using a prospective cohort study, Mah et al ${ }^{30}$ compared the decannulation rate, tolerance of oral diet, and hospital
LOS between a retrospective control group $(n=61)$, a group of subjects with tracheostomy whose care involved an intensivist-led tracheostomy service (tracheostomy service group, $n=124$ ), and a group of subjects with tracheostomy whose care involved using a tracheostomy bundle that consisted of an electronic postoperative tracheostomy order set, a tracking and following system, a decannulation protocol, and tracheostomy rounds by the intensivist-led tracheostomy service (post-tracheostomy care bundle group, $n=208$ ). The researchers noted that the decannulation rate before discharge $(26.0 \%)$ was significantly higher for the post-tracheostomy bundle group than the decannulation rates for the tracheostomy service group $(14.5 \%)$ and the control group $(8.2 \%)(P=.002)$. Additionally, the proportion of subjects in the post-tracheostomy bundle group that tolerated an oral diet before discharge (46.4\%) was significantly higher than the proportion of subjects in the tracheostomy service $(35.5 \%)$ or control groups $(24.6 \%)(P=$ $.005)$. There was no significant difference in the median hospital LOS between the 3 groups of subjects. Because this observational study of 393 subjects was conducted in a single institution and the severity of illness and duration of mechanical ventilation was different between groups, it is unclear whether these results can be generalized.

Over the past decade, the awareness of medical devicerelated pressure injury has increased, as have recommendations for preventive measures for reducing pressure injury caused by respiratory care devices, such as noninvasive positive pressure device interfaces and endotracheal tubes. ${ }^{34,35}$ Commensurate with the increasing vigilance in this area, O'Toole and colleagues ${ }^{29}$ investigated the association between a TRAPU bundle and hospital-acquired tracheostomy-related pressure ulcers using a pretest/posttest study of 338 subjects. The bundle used in the study 
consisted of 4 components: (1) placement of a hydrocolloid dressing underneath the tracheostomy flange in the postoperative period, (2) removal of plate sutures within $7 \mathrm{~d}$ of the tracheostomy procedure, (3) placement of a polyurethane foam dressing after suture removal, and (4) neutral positioning of the head. The researchers reported a significant reduction in the rate of hospital-acquired tracheostomyrelated pressure ulcers in the post-TRAPU care bundle group $(1.3 \%)$ compared to the pre-TRAPU care bundle group $(10.9 \%)(P<.001)$.

Given the benefits of using a structured approach to provide unique care to each patient, evidence supports the use of tracheostomy bundles that have been evaluated and approved by a team of individuals experienced in tracheostomy management for tracheostomized adult patients in the acute care setting (Evidence level B; median appropriateness score 7, range 6-8). This approach has been shown to decrease time to decannulation, decrease tracheostomy-related adverse events, and improve other tracheostomy-related outcomes, namely, tolerance of oral diet.

\section{Tracheostomy Teams}

The most rigorous assessment of the evidence regarding the use of tracheostomy teams in the management of patients with tracheostomy tubes was performed by Speed and Harding ${ }^{36}$ via a systematic review and meta-analysis. Because no randomized controlled trials had been conducted to investigate this issue, the researchers were able to include only 7 observational studies (pretest/posttest cohort design), which were of low to moderate quality. ${ }^{15-20,22}$ All 7 studies were single-center studies, and the composition of the tracheostomy team as well as the responsibilities and activities of the tracheostomy team were different across the studies. After the publication of this meta-analysis, 2 observational studies ${ }^{21,23}$ that focused on the use of a tracheostomy team were published. The study by Arora and colleagues ${ }^{14}$ was not included in the meta-analysis. These 3 additional observational studies were determined to be of low to moderate quality.

The outcomes on which the meta-analysis by Speed and Harding $^{36}$ focused were limited to time to decannulation and hospital LOS. However, other clinically important tracheostomy-related outcomes were reported in the metaanalysis. The results of the meta-analysis indicated that tracheostomy teams were associated with reductions in total tracheostomy time and increased speaking valve use. The authors cited insufficient evidence to determine the effect of tracheostomy teams on hospital or ICU LOS. Of the 10 studies $^{14-23}$ (all observational) that focused on the use of tracheostomy teams, 8 reported time to decannulation as an outcome, ${ }^{14-19,22,23}$ and 3 of these 8 studies $^{14,16,22}$ reported a significant decrease in time to decannulation in subjects managed by a tracheostomy team. Five studies reported LOS as an outcome, ${ }^{15,18,20-22}$ and these found significantly decreased LOS in subjects managed by a tracheostomy team; 1 study reported on ICU LOS, with no significant difference in ICU LOS in subjects managed by a tracheostomy team. ${ }^{16}$ Three studies ${ }^{15,17,18}$ reported speaking valve use as an outcome, with a significant increase in the use of speaking valves after tracheostomy team implementation. Six studies ${ }^{15-17,19-21}$ reported tracheostomy-related complications or adverse clinical events as an outcome, and all 6 studies reported significantly less adverse clinical outcomes in subjects managed by a tracheostomy team. One study reported tracheostomy-related cost, ${ }^{15}$ and 1 study reported tracheostomy tube downsizing time ${ }^{17}$ (Table 2).

The composition of the multidisciplinary tracheostomy team, role of team members, and team responsibilities varied for each study. In the 2 North American studies by de Mestral et $\mathrm{al}^{17}$ and Welton et $\mathrm{al},{ }^{23}$ a respiratory therapist was a member of the multidisciplinary team that included a physician and a speech-language pathologist. However, in other countries such as Australia and England, where respiratory therapists are unavailable, the multidisciplinary teams may consist of a combination of one or more physicians from different specialties (eg, otolaryngology, pulmonary, critical care), one or more nurses, a speech-language pathologist, a respiratory physiotherapist, a dietitian, and a social worker. Sodhi and colleagues ${ }^{21}$ used specially trained staff nurses for the specialized tracheostomy team.

Evidence supports the addition of a multidisciplinary tracheostomy team to the management strategy of tracheostomized adult patients in the acute care setting (Evidence level B; median score 7, range 5-8). A multidisciplinary tracheostomy team can decrease time to decannulation, LOS, and tracheostomy-related adverse events; this approach can also improve other tracheostomy-related outcomes, namely, increase in speaking valve use. The composition of the tracheostomy team should follow local custom.

\section{Protocol-Directed Care}

The use of evidence-based protocols in health care has become widely accepted, and respiratory therapists are expected to be highly proficient in the application of protocols to improve the quality of the care they provide. With regard to patients with tracheostomies, the inherent complexity of providing safe, efficacious care for them merits the use of evidence-based protocols. A review of the literature yielded a total of 5 studies $^{24-28}$ that focused on the use of protocols in the management of tracheostomized adult patients in the acute care setting. All 5 studies were observational and were judged to be of low to moderate quality. Three of the studies used a weaning/decannulation 
protocol, ${ }^{24,26,27}$ while 1 study used a swallow evaluation protocol in conjunction with a weaning/decannulation protocol, ${ }^{25}$ and 1 study compared 2 different swallow evaluation protocols. ${ }^{28}$

Most of the studies that investigated the effectiveness of protocols in the care of subjects with tracheostomies used time to decannulation as the primary outcome (Table 3). Frank and colleagues ${ }^{25}$ performed a retrospective chart review $3 \mathrm{y}$ after the implementation of a multidisciplinary swallowing and weaning protocol for dysphagic patients with tracheostomies. Data for 35 subjects who had tracheostomy tubes in situ after the protocol was implemented and data for 12 subjects with tracheostomy tubes in situ before the protocol was implemented were analyzed. The mean time to decannulation prior to protocol implementation was $94.7 \pm 60 \mathrm{~d}$; this time decreased to $48.2 \pm 51.6 \mathrm{~d}$ after implementation of the protocol $(P=.02)$. The researchers concluded that the multidisciplinary protocol was associated with earlier decannulation. In a prospective cohort study, Smith and colleagues ${ }^{26}$ compared time to decannulation between 3 different groups of subjects with tracheostomies: a baseline cohort that included patients who underwent tracheostomy before implementation of a decannulation protocol $(n=26)$, a pilot cohort that included subjects who underwent tracheostomy $1 \mathrm{y}$ after the protocol was implemented $(n=21)$, and a follow-up cohort that included subjects who underwent tracheostomy 2 y after the protocol was implemented $(n=39)$. The mean total time to decannulation in the baseline cohort was $15.5 \pm$ $12.1 \mathrm{~d}$. After pathway implementation in the pilot cohort, total time to decannulation decreased to $5.7 \pm 2.8 \mathrm{~d}(P<$ .001). In the follow-up cohort, total time to decannulation was $8.1 \pm 7.1 \mathrm{~d}(P=.003)$. These results demonstrated that the decannulation protocol was associated with decreased time to decannulation.

Clifford and Spencer ${ }^{24}$ used a pretest/posttest study involving 40 subjects to investigate the association between method of tracheostomy weaning and time to decannulation. They reported that implementation of a tracheostomy weaning protocol did not significantly decrease the time to decannulation $(4.2 \pm 4.23 \mathrm{~d}$ vs $2.85 \pm 1.35 \mathrm{~d})$. In a retrospective cohort study of 268 subjects, Thompson-Ward and colleagues ${ }^{27}$ compared the association between 2 different tracheostomy weaning protocols and time to decannulation. The results of the study indicated that the tracheostomy weaning protocol that did not include capping and routine downsizing in the decannulation assessment process was associated with a reduction in the time to tracheostomy tube decannulation from $30 \mathrm{~d}$ to $25 \mathrm{~d}(P<.05)$. Warnecke et $\mathrm{al}^{28}$ conducted a repeated-measures prospective observational study of 100 subjects with acute neurologic disease to determine the association between decannulation rate and type of swallow evaluation protocol. Each subject underwent a protocol-directed clinical swallow evaluation as well as a protocol-directed fiberoptic endoscopic evaluation of swallowing. The specific investigation (clinical swallow evaluation or fiberoptic endoscopic evaluation of swallowing) carried out by each investigator upon each subject was randomized to minimize order effects. The investigators found that tracheostomy tube decannulation decisions based on the fiberoptic endoscopic evaluation of swallowing protocol resulted in a greater number of subjects being decannulated $(54 \%)$ compared to decisions to decannulate based on clinical swallowing examination protocol (29\%).

Evidence supports the use of a weaning/decannulation protocol to guide weaning and removal of the tracheostomy tube in tracheostomized adult patients in the acute care setting (Evidence level B; median appropriateness score 8, range 8 ); this approach can improve time to decannulation.

\section{Summary}

Clinical decision-making regarding the various aspects of caring for patients with a tracheostomy is largely based on anecdotal evidence and the experience of individual care providers rather than on evidence-based guidelines. The complex nature of tracheostomy management, the heterogeneity of patients with tracheostomies, and the multifaceted outcomes linked to the care of these patients make it difficult to conduct randomized controlled trials. Consequently, we have endeavored to synthesize data from observational studies to complement the clinical consensus statement on tracheostomy care developed and promulgated by the American Academy of Otolaryngology-Head and Neck Surgery Foundation. ${ }^{37}$

One study indicated a higher rate of decannulation after the implementation of a tracheostomy management bundle. ${ }^{30}$ The tracheostomy bundle elements described in this study are based on scientific evidence as standardized order sets have been shown to reduce hospital LOS, mortality, and medication errors. ${ }^{38}$ Similarly, there is evidence that the electronic medical record system enhances the ability to identify and track specific patient populations. ${ }^{39,40}$

Our findings indicate that the use of specialized tracheostomy care teams is associated with decreased time to decannulation, less tracheostomy-related adverse events, and increased use of speaking valves. This finding is consistent with the results of the systematic review conducted by Speed and colleagues, ${ }^{36}$ which indicated that multi-disciplinary tracheostomy teams are associated with a mean reduction in total tracheostomy time of $8 \mathrm{~d}$ (95\% CI 6-11 d). A shorter time with the tracheostomy tube in situ confers many clinical benefits, including restoration of normal respiratory physiology, swallowing, cough function, and communication. Additionally, a shorter time with the tracheostomy tube in situ may reduce the risk for tracheostomy-related iatrogenic complications such as infection and death from an 
obstructed tube. It is unclear whether the differences in team composition, team member roles, and team responsibilities affected the outcome of the studies included in our systematic review.

The use of tracheostomy weaning and decannulation protocols can decrease the time to decannulation. This is consistent with the use of protocols in other areas of respiratory care. For example, there is strong evidence that the use of protocols in liberating patients from mechanical ventilation reduces the duration of mechanical ventilation. ${ }^{41}$ Other studies have reported that patients who are usually deemed difficult or impossible to wean from the tracheostomy tube were successfully decannulated after implementation of protocols. ${ }^{42}$

An important caveat with regard to the clinical outcomes of tracheostomized patients based on interventions is the underlying disease process that contributed to the need for tracheostomy insertion. Studies have noted that ICU and hospital LOS, time to decannulation, and time to oral intake commencement may vary based on underlying disease processes. ${ }^{43}$ The difference in etiologies for tracheostomy insertion must be taken into consideration when interpreting the effects of specific interventions on tracheostomyrelated clinical outcomes.

The evidence from multiple observational studies of low to moderate quality indicate that tracheostomy bundles, a multidisciplinary tracheostomy team, and tracheostomy weaning and decannulation protocols can reduce time to decannulation in tracheostomized adult patients in the acute care setting. Additionally, there is some evidence that a multidisciplinary tracheostomy team can reduce LOS and tracheostomy-related complications, but there is insufficient evidence regarding the effect of weaning and decannulation protocols on these outcomes. There is also insufficient evidence regarding the effect of tracheostomy teams and weaning/decannulation protocols on tracheostomy-related cost. More rigorously designed studies are needed to inform clinical decision-making in the care of tracheostomized adult patients in the acute care setting.

\section{REFERENCES}

1. Hess DR. Facilitating speech in the patient with a tracheostomy. Respir Care 2005;50(4):519-525.

2. Heffner JE, Miller KS, Sahn SA. Tracheostomy in the intensive care unit. Part 1: indications, technique, management. Chest 1986;90 (2):269-274.

3. Heffner JE, Miller KS, Sahn SA. Tracheostomy in the intensive care unit. Part 2: complications. Chest 1986;90(3):430-436.

4. Kollef MH, Ahrens TS, Shannon W. Clinical predictors and outcomes for patients requiring tracheostomy in the intensive care unit. Crit Care Med 1999;27(9):1714-1720.

5. Fischler L, Erhart S, Kleger GR, Frutiger A. Prevalence of tracheostomy in ICU patients: a nation-wide survey in Switzerland. Intensive Care Med 2000;26(10):1428-1433.
6. Frutos-Vivar F, Esteban A, Apezteguia C, Anzueto A, Nightingale P, Gonzalez M, et al. Outcome of mechanically ventilated patients who require a tracheostomy. Crit Care Med 2005;33(2):290-298.

7. Pierson DJ. Tracheostomy and weaning. Respir Care 2005;50(4):526533.

8. Adly A, Youssef TA, El-Begermy MM, Younis HM. Timing of tracheostomy in patients with prolonged endotracheal intubation: a systematic review. Eur Arch Otorhinolaryngol 2018;275(3):679-690.

9. Mehta AB, Syeda SN, Bajpayee L, Cooke CR, Walkey AJ, Wiener RS. Trends in tracheostomy for mechanically ventilated patients in the United States, 1993-2012. Am J Respir Crit Care Med 2015;192 (4):446-454.

10. Freeman BD, Stwalley D, Lambert D, Edler J, Morris PE, Medvedev $\mathrm{S}$, et al. High resource utilization does not affect mortality in acute respiratory failure patients managed with tracheostomy. Respir Care 2013;58(11):1863-1872.

11. Halum SL, Ting JY, Plowman EK, Belafsky PC, Harbarger CF, Postma GN, et al. A multi-institutional analysis of tracheotomy complications. Laryngoscope 2012;122(1):38-45.

12. Shah RK, Lander L, Berry JG, Nussenbaum B, Merati A, Roberson DW. Tracheotomy outcomes and complications: a national perspective. Laryngoscope 2012;122(1):25-29.

13. Mehta AB, Walkey AJ, Curran-Everett D, Douglas IS. One-year outcomes following tracheostomy for acute respiratory failure. Crit Care Med 2019;47(11):1572-1581.

14. Arora A, Hettige R, Ifeacho S, Narula A. Driving standards in tracheostomy care: a preliminary communication of the St Mary's ENT-led multidisciplinary team approach. Clin Otolaryngol 2008;33(6):596599.

15. Cameron TS, McKinstry A, Burt SK, Howard ME, Bellomo R, Brown DJ, et al. Outcomes of patients with spinal cord injury before and after introduction of an interdisciplinary tracheostomy team. Crit Care Resusc 2009;11(1):14-19.

16. Cetto R, Arora A, Hettige R, Nel M, Benjamin L, Gomez CMH, et al. Improving tracheostomy care: a prospective study of the multidisciplinary approach. Clin Otolaryngol 2011;36(5):482-488.

17. de Mestral C, Iqbal S, Fong N, LeBlanc J, Fata P, Rzaek T, Khwaja K. Impact of a specialized multidisciplinary tracheostomy team on tracheostomy care in critically ill patients. Can J Surg 2011;54(3):167172.

18. LeBlanc J, Shultz JR, Seresova A, de Guise E, Lamoureux J, Fong N, et al. Outcome in tracheostomized patients with severe traumatic brain injury following implementation of a specialized multidisciplinary tracheostomy team. J Head Trauma Rehabil 2010;25(5):362-365.

19. Norwood MGA, Spiers P, Bailiss J, Sayers RD. Evaluation of the role of a specialist tracheostomy service. From critical care to outreach and beyond. Postgrad Med J 2004;80(946):478-480.

20. Parker V, Giles M, Shylan G, Austin N, Smith K, Morison J, Archer W. Tracheostomy management in acute care facilities-a matter of teamwork. J Clin Nurs 2010;19(9-10):1275-1283.

21. Sodhi K, Shrivastava A, Singla MK. Implications of dedicated tracheostomy care nurse program on outcomes. J Anesth 2014;28(3):374-380.

22. Tobin AE, Santamaria JD. An intensivist-led tracheostomy review team is associated with shorter decannulation time and length of stay: a prospective cohort study. Crit Care 2008;12(2):R48.

23. Welton C, Morrison M, Catalig M, Chris J, Pataki J. Can an interprofessional tracheostomy team improve weaning to decannulation times? A quality improvement evaluation. Can J Respir Ther 2016;52(1):7-11.

24. Clifford C, Spencer A. An evaluation of the impact of a tracheostomy weaning protocol on extubation time. Nurs Crit Care 2009;14(3):131138.

25. Frank U, Mäder M, Sticher H. Dysphagic patients with tracheotomies: a multidisciplinary approach to treatment and decannulation management. Dysphagia 2007;22(1):20-29. 


\section{Management of Adult Tracheostomy}

26. Smith KA, Matthews TW, Dubé M, Spence G, Dort JC. Changing practice and improving care using a low-risk tracheotomy clinical pathway. JAMA Otolaryngol Head Neck Surg 2014;140 (7):630-634 .

27. Thompson-Ward E, Boots R, Frisby J, Bassett L, Timm M. Evaluating suitability for tracheostomy decannulation: a critical evaluation of two management protocols. J Med Speech-Lang Patho 1999;7 (4):273-281.

28. Warnecke T, Suntrup S, Teismann IK, Hamacher C, Oelenberg S, Dziewas R. Standardized endoscopic swallowing evaluation for tracheostomy decannulation in critically ill neurologic patients. Crit Care Med 2013;41(7):1728-1732.

29. O'Toole TR, Jacobs N, Hondorp B, Crawford L, Boudreau LR, Jeffe $\mathrm{J}$, et al. Prevention of tracheostomy-related hospital-acquired pressure ulcers. Otolaryngol Head Neck Surg 2017;156(4):642-651.

30. Mah JW, Staff II, Fisher SR, Butler KL. Improving decannulation and swallowing function: a comprehensive, multidisciplinary approach to post-tracheostomy care. Respir Care 2017;62(2):137-143.

31. Fitch K, Bernstein SJ, Aguilar MD, Burnand B, LaCalle JR, Lazaro P, et al. The RAND/UCLA Appropriateness Method User's Manual. Santa Monica, CA: RAND; 2001.

32. Ospina MB, Mrklas K, Deuchar L, Rowe BH, Leigh R, Bhutani M, Stickland MK. A systematic review of the effectiveness of discharge care bundles for patients with COPD. Thorax 2017;72(1):31-39.

33. Klompas M, Li L, Kleinman K, Szumita PM, Massaro AF. Associations between ventilator bundle components and outcomes. JAMA Intern Med 2016;176(9):1277-1283.

34. Black JM, Cuddigan JE, Walko MA, Didier LA, Lander MJ, Kelpe MR. Medical device related pressure ulcers in hospitalized patients. Int Wound J 2010;7(5):358-365.
35. Mussa CC, Meksraityte E, Li J, Gulczynski B, Liu J, Kuruc A. Factors associated with endotracheal tube related pressure injury. SM J Nurs 2018;4(1):1-6.

36. Speed L, Harding KE. Tracheostomy teams reduce total tracheostomy time and increase speaking valve use: a systematic review and metaanalysis. J Crit Care 2013;28(2):216.e1-e10.

37. Mitchell RB, Hussey HM, Setzen G, Jacobs IN, Nussenbaum B, Dawson $\mathrm{C}$, et al. Clinical consensus statement: tracheostomy care. Otolaryngol Head Neck Surg 2013;148(1):6-20.

38. Wells C, Loshak H. Standardized Hospital Order Sets in Acute Care: A Review of Clinical Evidence, Cost-Effectiveness, and Guidelines. Ottawa, ON: Canadian Agency for Drugs and Technologies in Health; 2019.

39. LaRoché KD, Hinkson CR, Thomazin BA, Minton-Foltz PK, Carlbom DJ. Impact of an electronic medical record screening tool and therapist-driven protocol on length of stay and hospital readmission for COPD. Respir Care 2016;61(9):1137-1143.

40. Pollack AH, Kronman MP, Zhou C, Zerr DM. Automated screening of hospitalized children for influenza vaccination. J Pediatric Infect Dis Soc 2014;3(1):7-14.

41. Blackwood B, Alderdice F, Burns K, Cardwell C, Lavery G, O'Halloran P. Use of weaning protocols for reducing duration of mechanical ventilation in critically ill adult patients: Cochrane systematic review and meta-analysis. BMJ 2011;342(jan13 2):c7237.

42. Gundogdu I, Ozturk EA, Umay E, Karaahmet OZ, Unlu E, Cakci A. Implementation of a respiratory rehabilitation protocol: weaning from the ventilator and tracheostomy in difficult-to-wean patients with spinal cord injury. Disabil Rehabil 2017;39(12):1162-1170.

43. Pryor L, Ward E, Cornwell P, O'Connor S, Chapman M. Patterns of return to oral intake and decannulation post-tracheostomy across clinical populations in an acute inpatient setting. Int J Lang Commun Disord 2016;51(5):556-567. 